Gerión. Revista de Historia Antigua

ISSN: 0213-0181

\title{
Priscilliano e il suo Simbolo: un caso di studio sui Simboli nella Chiesa occidentale durante la seconda metà del quarto secolo ${ }^{1}$
}

Kouichi Hayashi

Recibido: 16 de febrero de 2021 / Aceptado: 19 de abril de 2021

Sommario. I Simboli sono elemento importante delle controversie sulla dottrina nella Chiesa antica. Questo articolo tratta dell'usa e del testo di un Simbolo che Priscilliano testimoniò, quello che fu condannato e giustiziato come mago ed eretico, come Mani, alla fine del IV secolo. Il suo Simbolo non era un'invenzione e la sua interpretazione della parola symbolum non era strana, ma era un testimone della situazione in cui i Simboli erano usati nell'antica Chiesa iberica.

Parola chiave: nomi per il credo; Simbolo degli Apostoli; Simbolo niceno; Chiesa iberica antica.

\section{[en] Priscillian of Avila and His Creed: A Case Study on the Creeds in the Western Church during the Second Half of $4^{\text {th }}$ Century}

Abstract. Creeds are an important element of the controversies over the doctrine in the ancient Church. This article deals with the usage and the text of a creed which Priscillian testified, who was condemned and executed as a sorcerer and heretic like Mani in the late $4^{\text {th }}$ century. His creed was not an invention and his interpretation of the word symbolum was not strange, he was rather a witness to the situation in which the creeds were used in the ancient Iberian Church.

Keywords: Names for the Creed; Apostles' Creed; Nicene Creed; Ancient Iberian Church.

Sommario: 1. Introduzione. 2. Priscilliano di Avila. 2.1. Profilo di Priscilliano. 2.2. Lo status quaestionis. 3. Il Simbolo di Priscilliano. 3.1. Le fonti e il testo del Simbolo. 3.2. Il simbolo secondo Priscilliano. 4. Il Simbolo di Priscilliano nella seconda metà del quarto secolo. 4.1. Il senso di symbolum. 4.2. Confronto con il Simbolo degli Apostoli. 4.3. Simbolo degli Apostoli e Simbolo niceno nella Chiesa occidentale durante la seconda metà del quarto secolo. 5. Conclusione. 6. Bibliografia.

Cómo citar: Hayashi, K. (2021): Priscilliano e il suo Simbolo: un caso di studio sui Simboli nella Chiesa occidentale durante la seconda metà del quarto secolo, en Gerión 39(2), 523-539.

\footnotetext{
1 Questo articolo è sviluppato da Hayashi 2018 [in giapponese]. Ringrazio a The Japanese Society for Patristic Studies per il permesso di rinnovarlo, e anche a Prof.ssa Veronese per la consulenza benevola.

2 Keio University.

E-mail: swtk.niki@gmail.com

ORCID: 0000-0001-7110-0883
} 


\section{Introduzione}

La crisi ariana è un evento così esteso nel Cristianesimo antico che determinò lo sviluppo della dottrina trinitaria. La controversia sul dogma coinvolse anche il Simbolo, poiché approfondendo la conoscenza della relazione tra Padre e Figlio i partiti si contrapposero sulle espressioni dei Simboli approvati dai concili. ${ }^{3}$ La fede del Concilio di Nicea fu riconosciuta come la fede ortodossa, anche se solo dopo un lungo processo in quanto il Simbolo niceno-costantinopolitano non fu accolto subito e uniformemente dalle Chiese, così come in ritardo fu introdotto nella liturgia e in Occidente dove perdurava l'uso del Simbolo degli Apostoli. ${ }^{4}$ Come erano usati questi Simboli nella seconda metà del quarto secolo quando stava per essere stabilita la fede ortodossa rappresentata dal simbolo?

Intendo affrontare questa questione trattando il Simbolo di Priscilliano come un caso di studio per verificare qual è il testo del suo Simbolo e come ha compreso questo Simbolo. La struttura dell'analisi è la seguente: (1) profilo di Priscilliano e status quaestionis sul suo Simbolo, (2) analisi del Simbolo di Priscilliano, (3) Simbolo di Priscilliano alla luce del contesto coevo.

\section{Priscilliano di Avila}

\subsection{Profilo di Priscilliano}

Non c'è quasi niente che sappiamo con certezza sulla sua formazione e la nostra conoscenza della sua attività è limitata agli ultimi cinque anni della sua vita. ${ }^{5} \mathrm{E}$ il Concilio di Saragozza (380) il primo evento che collega il nome di Priscilliano con una data concreta. ${ }^{6}$ In conseguenza di questo concilio, elevandosi il livello del conflitto tra i priscillianisti ${ }^{7}$ e i suoi oppositori, Idazio di Merida fece una petizione alla corte a Milano e riuscì a far applicare ai priscillianisti una legge contro l'eresia. ${ }^{8}$ Bandito dalla Spagna, Priscilliano partì per Italia e difese la propria innocenza davanti ai rappresentanti della Chiesa e della corte imperiale. Non riuscì a ottenere il sostegno di Papa Damaso e di Ambrogio, ma attraverso l'aiuto di un funzionario a corte fu abrogato il riscritto che aveva ordinato il suo esilio, e così furono gli antipriscillianisti a ritrovarsi nella posizione di imputati. ${ }^{9}$ Tuttavia la sorte era cieca: nel 383 Magno Massimo fu proclamato imperatore in Britannia e conquistò la prefettura

3 Kelly 1972, 205-207, 263-264. Tutte le date in questo articolo sono A.D. salvo diversa indicazione.

4 Non è sicuro il tempo della sua introduzione nell'Eucaristia. Cf. Kinzig 2017, 1.10. Kinzig (2011, 63-99) dimostra che una delle forze per confermare il Simbolo nella Chiesa fu l'istituzione delle feste durante la fine del IV secolo e l'inizio del V secolo.

5 Generalmente si accetta 1'idea che Priscilliano proveniva da una famiglia ricca e potente, forse senatoriale, e ricette una formazione appropriata alla sua origine. Cf. Stroheker 1963, 118-119. Sulla data di nascita e sulla patria, Vollmann 1974, 490-493; Sanchez 2009, 29, n. 2.

6 Sanchez $(2009,32)$ ha difeso la nuova teoria che lo colloca all'anno 379, tuttavia seguo l'opinione diffusa. Cf. Burrus 1995, 190-191, n. 36.

7 Quanto alle fonti esistenti, il nome Priscillianistae come una vera e propria setta appare per la prima volta dopo la morte di Priscilliano. La prima comparsa è nella legge nel 407: Cod. Theod. 16.5.40 (Mommsen - Rougé 2005). Cf. Sanchez 2009, 93. Tuttavia chiamo priscillianisti Priscilliano e i suoi seguaci in quel tempo per convenienza.

8 Sulp. Sev. Chron. 2.47.1-2 (De Senneville-Grave 1999); Priscill. Tract. 2.39.17-41.2 (Schepss 1889).

$9 \quad$ Sulp. Sev. Chron. 2.48.1-49.2 (De Senneville-Grave 1999). 
del pretorio delle Gallie vincendo Graziano. ${ }^{10}$ Il nuovo imperatore diede ascolto agli anti-priscillianisti e ordinò di convocare un concilio a Bordeaux e di citare Priscilliano e i suoi seguaci. Priscilliano non si presentò, ma a Treviri fu giudicato e condannato alla pena di morte da un tribunale secolare. ${ }^{11}$

Priscilliano in vita era caduto in sospetto di maleficio e di manicheismo, ${ }^{12}$ in aggiunta dopo la sua morte fu imputato di diverse eresie ed errori dai Padri. Di lui scrissero Padri, come Gerolamo, ${ }^{13}$ Sulpicio Severo, ${ }^{14}$ Orosio, ${ }^{15}$ Agostino ${ }^{16}$ e Leone Magno, ${ }^{17}$ e nei secoli successivi si replicò l'immagine di Priscilliano costruita sulla base delle loro testimonianze. Fino alla fine del XIX secolo non vi fu alcuno sviluppo su Priscilliano e sul priscillianismo, poiché la ricerca si basava solo sulle notizie ricavate dai Padri. ${ }^{18}$

\subsection{Lo status quaestionis}

Alla fine di XIX secolo una scoperta rivoluzionò la situazione. G. Schepss attribuì a Priscilliano undici scritti anonimi contenuti in un manoscritto che aveva scoperto nella biblioteca universitaria di Würzburg e ne pubblicò l'edizione nel $1889 .{ }^{19}$ Questi scritti, detti Tractatus di Würzburg, hanno rivelato una nuova immagine di Priscilliano demolendo quella formata delle notizie dei Padri.

Nei decenni successivi furono fornite diverse interpretazioni su Priscilliano e sul movimento radunato intorno a lui. Uno studioso spiegò il priscillianismo come fenomeno economico-sociale e nazionale. ${ }^{20}$ Tuttavia gli studi principali lo hanno interpretato dal punto di vista spirituale. Negli anni Settanta del secolo scorso B. Vollmann e H. Chadwick pubblicarono i loro studi, in cui valutarono il pensiero di Priscilliano non come un'eresia manichea o gnostica, ma Priscilliano stesso come un carismatico o una persona dedita all'occultismo. ${ }^{21} \mathrm{E}$ così, recentemente gli studi principali collocano Priscilliano nel contesto più ampio del cristianesimo coevo, evitando il semplice schema 'ortodossa-eresia'. Particolare era considerata la sua comprensione monarchiana della Trinità così come la dottrina dualistica. Già da tempo la critica aveva individuato la tendenza monarchiana dei Tractatus, ${ }^{22}$ ma nel XXI secolo la controversia intorno Priscilliano è stata spiegata alla luce della crisi ariana in Occidente. M. V. Escribano Paño ha ipotizzato che il modello dei priscillianisti furono i luciferiani, rigoristi della fede nicena che derivano il loro nome da Lucifero di Cagliari, ${ }^{23}$ e che la controversia si comprende come l'estensione del conflitto tra

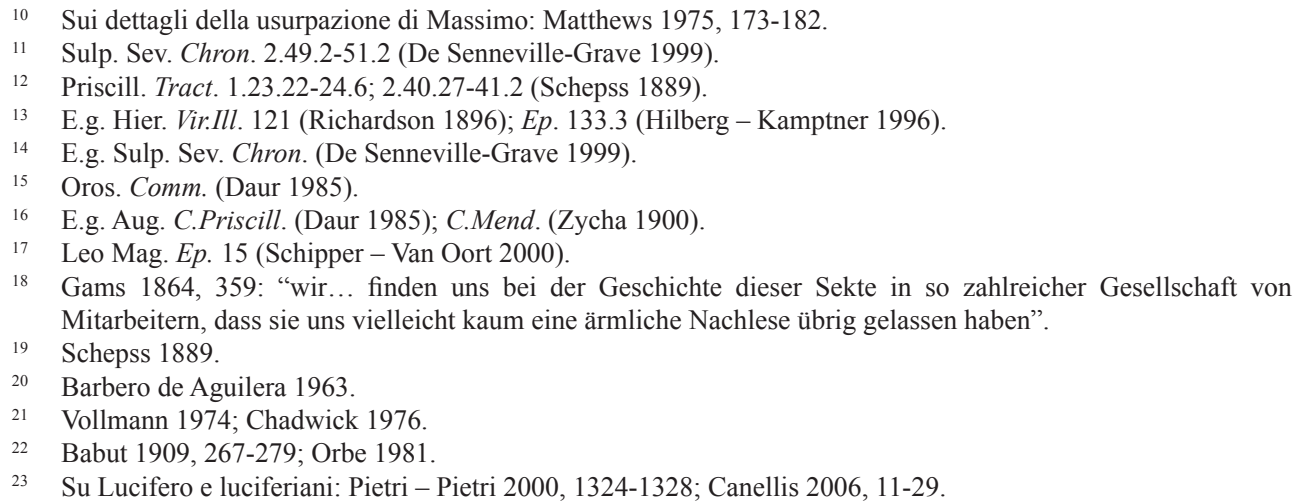


rigoristi e moderati sul trattamento dei vescovi che hanno sottoscritto il simbolo semiariano al concilio di Rimini. ${ }^{24}$

Come ho riferito nell'Introduzione, il Simbolo è un importante elemento nell'ambito della controversia ariana, e anche il Simbolo di Priscilliano è stato valutato dagli studiosi dal punto di vista della sua conoscenza trinitaria. La confusione di persone era stata sottolineata fin dall'inizio del XX secolo ${ }^{25} \mathrm{e}$ anche gli studiosi del Simbolo trovavano elementi particolari nel Simbolo di Priscilliano. ${ }^{26}$ Contro questa posizione $\mathrm{T}$. Toom ha supposto che questi caratteri fossero compresi come misure per contrastare l'arianesimo, approvando la spiegazione di Escribano Paño. ${ }^{27}$ D'altra parte N. McLynn ha giudicato il Simbolo priscillianista come una rappresentazione di carattere locale isolato di cristianesimo spagnolo in quell'epoca. ${ }^{28}$

Le opinioni sul Simbolo di Priscilliano si dividono così come quelle sul suo pensiero trinitario. Passiamo quindi ad esaminare il Simbolo stesso.

\section{Il Simbolo di Priscilliano}

\subsection{Le fonti e il testo del Simbolo}

Il Simbolo di Priscilliano è contenuto nel Liber ad Damasum episcopum (=Tract. 2), uno dei Tractatus di Würzburg. Nonostante ci siano tante discussioni sulla paternità degli undici scritti, si riconosce generalmente che questa opera è di Priscilliano stesso oppure di un suo compagno, perché in essa ci sono riferimenti a nomi o a eventi che trovano riscontro in altre fonti..$^{29}$ Inoltre si può determinare la data approssimativa della sua composizione. Come è stato detto prima, durante il viaggio verso l'Italia Priscilliano provò a essere ricevuto dalle autorità ecclesiastiche, tra cui papa Damaso, e imperiali, pertanto si considera che lo scritto fu composto nel 381 oppure nel $382 .{ }^{30}$

Questa opera è una tipica petizione, da cui si evince l'oratoria tattica dell'autore. ${ }^{31}$ Schepss individuò nel testo tre fasi: 1 . controbattendo 1'accusa, l'autore insiste che lui e i suoi amici seguono la fede ortodossa e che è ingiusta la denuncia fatta da Idazio (34.3-37.17); 2. successivamente, condanna le eresie come l'arianesimo, il patripassianesimo, il novatianismo, il manicheismo (37.1839.16); 3. infine, tornando al suo argomento, racconta gli eventi successi dopo il concilio di Saragozza e chiude con la supplica di farsi il giusto giudizio sotto la autorità della sede apostolica.

\footnotetext{
Escribano Paño 2005.

Künstle 1905, 21.

Kelly 1972, 178; Westra 2002, 146.

Toom 2014a; 2014b.

McLynn 1997.

29 Si considerano così non solo Liber ad Damasum episcopum (Tract. 2) ma anche due opere: Liber apologeticus (Tract. 1), Liber de fide et de apocryphis (Tract. 3), che uso come materia per il mio argomento. Sulla discussione sulla paternità dei Tractatus di Würzburg: Chadwick 1976, 63-69; Conti 2010, 14-17; Veronese a questo stesso volume.

30 Sanchez 2009, 72-73.

31 Van Waarden 2014, 138-141.
} 
Nella prima parte c'è il Simbolo di Priscilliano. ${ }^{32}$ Uno dei caratteri del Simbolo sono le citazioni della Bibbia introdotte dalla formula sicut scribtum est, da cui si ricava il testo:

Art. 1. Credentes unum deum patrem omnipotentem

Art. 2. et unum dominum Iesum Christum

Art. 3. natum ex Maria virgine ex spiritu sancto

Art. 4. passum sub Pontio Pilato, crucifixum

Art. 5. sepultum, tertia die resurrexisse

Art. 6. ascendisse in caelos, sedere ad dexteram dei patris omnipotentis

Art. 7. inde venturum et iudicaturum de vivis et mortuis

Art. 8. credentes in sanctam eclesiam, sanctum spiritum, baptismum salutare

Art. 9. credentes remissionem peccatorum

Art. 10. credentes in resurrectionem carnis

Prima di fare l'analisi e il confronto del testo, mostro come Priscilliano comprende il Simbolo stesso.

\subsection{Il simbolo secondo Priscilliano}

È chiaro che Priscilliano ha chiamato questo testo il Simbolo: symbolum nella citazione seguente:

Custodendo la via di questo simbolo tutte le dottrine, gli istituti e i dogmi delle eresie, che si disputarono a causa non di ingegno ma di faziosità, condanniamo con la professione ortodossa e battezziamo, com'è scritto, nel nome del Padre, del Figlio e dello Spirito Santo. ${ }^{33}$

Successivamente insistendo sull'unità di Dio comincia la serie delle condanne, in cui afferma: "se cadiamo nella situazione che condanniamo, siamo condannati dalla stessa professione dei nostri libretti". ${ }^{34}$ Anche all' inizio dell'opera è citata la fede ortodossa osservata dal simbolo ${ }^{35}$ dunque secondo Priscilliano il simbolo è la rappresentazione della fede ortodossa. ${ }^{36}$ Priscilliano dà testimonianza anche

32 Tract. 2.36.14-37.17 (Schepss 1889).

33 Tract. 2.37.18-21 (Schepss 1889): Cuius symboli iter custodientes omnes hereses doctrinas instituta uel dogmata, quae sibi altercationem non ingenia, sed studia fecerunt, catholico ore damnamus, baptizantes, sicut scribtum est, in nomine patris et fili et spiritus sancti.

34 Tract. 2.38.6-7 (Schepss 1889): si in ea quae damnamus incurrimus, ipsa libelli nostri professione damnemur.

35 Tract. 2.34.3 (Schepss 1889): catholica fides dati per deum symboli iter possidens.

36 Ho tradotto la parola catholicus con "ortodosso" o "la fede ortodossa", oppure "universalmente". Qui non ho intenzione di esaminare in generale l'uso di questa parola nel mondo latino a quel tempo, ma di verificarne l'uso in Priscilliano. Inizialmente è chiaro che Priscilliano usa questa parola come l'espressione contraria a heresis o hereticus. E.g. Tract. 1.6.19-20; Tract. 2.38.7-8; Tract. 2.42.8-9; Tract. 3.51.2526 (Schepss 1889). Quindi come è definita l'eresia da Priscilliano? Come indica l'iniziale citazione dal Tract. 3, è una invenzione nuova che devia dalla tradizione osservata universalmente in chiesa. E.g. Tract. 3.44.3-5; Tract. 1.23.9-18. Priscilliano non ha mai il dubbio di non essere catholicus e parte di questa ortodossia. In effetti non definisce sé stesso come un'altra setta separata dalla chiesa (Tract. 2.39.7-8). In aggiunta insiste che siano fedeli alla tradizione della chiesa e che si deve condannare ogni deviazione da questa (Tract. 2.41.23-42.3). Penso pertanto che Priscilliano comprenda la ortodossia non come la dottrina 
della situazione in cui si usa il simbolo. Nella citazione all'inizio di questa sezione, si parla della condanna dell'eresia e successivamente si parla del battesimo; in altra occasione si collegano chiaramente il simbolo e il battesimo, dicendo:

Infatti Cristo Dio, il Figlio di Dio, che ha patito nella carne, è tutta la fede, tutta la vita e tutta la venerazione per noi che siamo stati battezzati secondo la fede del simbolo e eletti al sacerdozio nel nome del Padre, del Figlio e dello Spirito Santo. ${ }^{37}$

Nel Liber apologeticus (=Tract. 1) non è citato il testo del simbolo, ma si dice: “e rifiutando i desideri della vita precedente di cui ci vergognavamo, abbiamo accolto alla via della grazia rinnovata il simbolo universalmente osservato che teniamo" ${ }^{38} \mathrm{e}$ "tuttavia noi entrati attraverso il simbolo nel corpo di Dio, cioè nella chiesa venerabile, riconosciamo nella disposizione dei quattro Vangeli la fede indissolubile e irrigata dall'unica fonte trina", ${ }^{39}$ dove si mette il simbolo in relazione con l'iniziazione. Ma la più importante testimonianza è in Liber de fide et de apocryphis:

La scritta di Dio è cosa solida, cosa vera, non eletta dagli uomini ma data da Dio agli uomini, di cui se una parte è santa anche il tutto è santo. Dunque [appare] l'eresia, quando ognuno serve la sua opera piuttosto che Dio e non segue il simbolo ma ha intenzione di discutere intorno al simbolo, perché non terrebbe niente oltre al simbolo se conoscesse la fede. Esattamente il symbolum è il sigillo della cosa vera e distruggere (de-signare $)^{40} \mathrm{il} \mathrm{simbolo} \mathrm{è} \mathrm{discutere} \mathrm{intorno} \mathrm{al} \mathrm{simbolo} \mathrm{piuttosto}$ che credere ad esso. Il symbolum è l'opera di Dio nel nome del Padre, del Figlio e dello Spirito Sancto, la fede in un solo Dio, da cui è nato Cristo, Dio, figlio di Dio e Salvatore, che ha patito nella carne, è risorto per l'amore verso gli uomini, dando il simbolo ai suoi apostoli. ${ }^{41}$

In questo passo si insiste sul fatto che il simbolo sia il criterio della fede più chiaramente che nei riferimenti del Liber ad Damasum episcopum. L'autore dice che alterare la tradizione rappresenta l'inizio delle eresie così come inserire nuove aggiunte tanto al simbolo quanto alla Bibbia; sostiene inoltre che il simbolo sia

definita dalla chiesa specifica o dal concilio ecumenico, ma come la fede secondo la tradizione provenuta dagli Apostoli e conservata in tutte le chiese. Non ho tradotto la parola catholicus letteralmente solo in "ortodosso" tenendo in conto la sua accezione di universalità.

37 Tract. 2.39.13-16 (Schepss 1889): Nobis enim Christus deus dei filius passus in carnem secundum fidem symboli baptizatis et electis ad sacerdotium in nomine patris et fili et spiritus sancti tota fides, tota vita, tota veneratio est.

38 Tract. 1.4.17-5.1 (Schepss 1889): et repudiatis prioris vitae desideriis, in quibus erubescebamus, ad innovatae iter gratiae simbolum catolicae observationis accepimus, quod tenemus.

39 Tract. 1.31.28-31 (Schepss 1889): Nos autem venerabilis eclesiae dei per symbolum corpus ingressi indissolubilem fidem uno fonte tripertito rigatam in quattuor evangeliorum dispositione cognovimus.

40 Priscilliano usò caratteristicamente questo verbo, il quale significa "rompere un sigillo". Cf. Blaise 1967, 261.

${ }_{41}$ Tract. 3.48.17-49.6 (Schepss 1889): Scribtura dei res solida, res vera est nec ab homine electa, sed homini de deo tradita, cuius si dilibatio sancta est et massa sancta est. Inde denique heresis, dum singuli quique ingenio suo potius quam deo serviunt et non sequi symbolum, sed de symbolo disputare disponunt, cum, sifidem nossent, extra symbolum nil tenerent. Symbolum enim signatura rei verae est et designare symbolum est disputare de symbolo malle quam credere; symbolum opus domini est in nomine patris et fili et spiritus sancti, fides unius dei, ex quo Christus deus dei filius salvator natus in carne passus resurrexit propter hominis amorem; qui apostolis suis symbolum tradens. 
trasmesso per tradizione da Cristo attraverso gli Apostoli. Pertanto Priscilliano ha usato lo stesso simbolo che aveva ottenuto per il battesimo contro la denuncia che ha messo in dubbio la sua fede cristiana. ${ }^{42}$ A questo punto provo a collocare il Simbolo di Priscilliano e la sua conoscenza del simbolo nel contesto in quel tempo.

\section{Il Simbolo di Priscilliano nella seconda metà del quarto secolo}

\subsection{Il senso di symbolum}

Nel quarto secolo è usuale sottolineare che il simbolo battesimale ha origine da Cristo e dagli apostoli. ${ }^{43}$ La stessa parola symbolum era un termine inizialmente utilizzato per il simbolo battesimale e dopo il quinto secolo si è usato anche per il simbolo dei concili. ${ }^{44}$ Il termine greco $\sigma u ́ \mu \beta o \lambda o v$ è all'origine del latino symbolum, ma il suo uso come "simbolo" prende origine dal termine latino. ${ }^{45}$ Tuttavia non è chiaro perché la parola symbolum sia diventata il termine per indicare il simbolo. È sicuro che Tertulliano la usava ${ }^{46}$ e Cipriano ha indicato con essa l'interrogazione battesimale. ${ }^{47}$ Ma non sono unanimi le opinioni dei Padri riguardo all'origine del termine e pure gli studiosi forniscono diverse spiegazioni. ${ }^{48} \mathrm{~W}$. Kinzig ha suddiviso le diverse interpretazioni del termine da parte dei Padri in cinque categorie: ${ }^{49} 1$. il segno (token) (a) per riconoscere i cristiani, (b) della piena conoscenza della verità, (c) della giusta fede; 2. la sintesi (summary) della fede cristiana; 3. il ricordo (reminder) della fede e della confessione santa; 4. il contratto (contract) (a) tra credenti, (b) dei singoli credenti con Dio; 5. il segno, il simbolo (sign, symbol). L'ultima interpretazione è quella di Priscilliano, riguardo alla quale Kinzig ha sostenuto: "this interpretation of symbolum as signatura rei verae is only found in Priscillian. The res vera is the Holy Scripture to which the symbolum refers". ${ }^{50}$ A prima vista sembra un giudizio che considera Priscilliano un eretico. Ma mi contrappongo a questa spiegazione.

La questione riguarda il senso di signatura rei uerae. Concordo con Kinzig su un punto: che res vera significa la Bibbia, perché Priscilliano ha detto: "La scrittura di Dio è cosa solida e cosa vera, non è eletta dagli uomini ma consegnata agli uomini da Dio". ${ }^{51}$ Tuttavia ho un'opinione contraria rispetto a Kinzig sul significato di signatura. Considero che questa parola indichi non il segno simbolico ma concreto, cioè il sigillo. Priscilliano non l'ha usata in nessun altro luogo e non ci sono tanti esempi nei dizionari, ${ }^{52}$ però 1 'impiego antico dimostra il significato di "matrice del

42 Il simbolo battesimale ha l'origine più antica, ma la formulazione di simbolo dal concilio è nuovo fenomeno dopo il quarto secolo. Gli studiosi del simbolo affermano che la funzione di simbolo si amplia grazie alla controversia sulla dottrina. Kelly 1972, 205-211; Westra 2002, 42-43; Kinzig 2011, 7-9.

43 Ambr. Symb. 2 (Botte 1994); Rufin. Symb. 2 (Simonetti 1961).

44 Westra 2010, 91.

45 Kelly 1972, 52-53; Kinzig 2011, 3-7.

46 Tert. Paenit. 6.12 (Munier 1984); Adv.Marc. 5.1.2 (Moreschini - Braun 2004).

47 Cypr. Ep. 69.7.1-2 (Diercks 1996).

48 Kelly 1972, 53-56; Westra 2010, 85-86.

49 Kinzig 2017, 1.5-6.

50 Kinzig 2017, 1.6.

51 Tract. 3.48.17-18 (Schepss 1889): Scribtura dei res solida, res vera est nec ab homine electa, sed homini de deo tradita.

52 Per esempio, non c' è il lemma in Niermeyer - Van de Kieft 2002; Gaffiot - Flobert 2000; Blaise 1967. 
sigillo" 53 e di "marchiatura" 54 e nel latino medievale di "firma". ${ }^{55}$ Nonostante il mutamento di significato da sigillo o da segno del sigillo fino all'identificazione con firma, comunque si intende l'atto di mettere un sigillo.

Questo ipotesi trova fondamento nelle espressioni per il battesimo. A volte il verbo signare esprime il lavoro dello Spirito Santo nella Bibbia, ${ }^{56} \mathrm{e}$ Tertulliano usa le parole come obsignare, signare, obsignatio e signaculum quando parla del battesimo, soprattutto in relazione alla immersione con la interrogazione battesimale. ${ }^{57}$ Così mettendo in conto il collegamento tra il battesimo e il sigillo, sarebbe naturale l'uso di un termine relativo al sigillare per definire il simbolo battesimale. Infatti, Ambrogio afferma:

Ora è per noi il tempo e il giorno prestabilito di consegnare il Simbolo; il quale Simbolo è un contrassegno dello Spirito; il quale Simbolo è la meditazione del nostro cuore ed è come un baluardo sempre presente; senza dubbio è il tesoro del nostro spirito. ${ }^{58}$

Poi Rufino sostiene: "Io, come lui [Girolamo] e tutti sanno, trovandomi nel monastero già prima di trent'anni, rigenerato dalla grazia del battesimo, ho conseguito il segno (signaculum) della fede per opera dei beati Cromazio Giovino ed Eusebio". ${ }^{59}$ Siccome successivamente aggiunge che Eusebio è "per me padre e maestro del simbolo e della fede" ${ }^{60}$ e sembra che i testi che spiega seguono il testo di Simbolo degli Apostoli, si potrebbe dire che anche Rufino consideri il Simbolo come sigillo. Mi sembra che Priscilliano consideri il Simbolo come il sigillo di cosa vera ritenendo che la fede nella Bibbia sia condensata nel simbolo, ispirandosi al ricordo e alla confessione del simbolo nel battesimo. È un giudizio affrettato sostenere che l'interpretazione di Priscilliano sia isolata e bizzarra ${ }^{61}$ perché si deve tradurre la parola signatura con sigillo secondo gli esempi contemporanei.

\footnotetext{
At. Cap. De pontificio iure 10 (Kübler - Seckel 1908).

Pallad. Agric. 5, tituli VI; 5.6 (Hodgers 1975).

ASS. 17 Junii (Carnandet 1867, 323).

2 Cor 1, 22; Eph 1, 13.

57 E.g. obsignare: Pudic. 9.16 (Munier 1993); Bapt. 6.1 (Borleffs 1954); Idol. 12.1 (Waszink - Van Winden 1987); obsignatio: Bapt. 13.2; Paenit. 6.16 (Munier 1984); signare: Adv.Marc. 1.28.3 (Braun 1990); Praescr. 36.5 (Refoulé - De Labriolle 1957); signaculum: Pudic. 9.11 (Munier 1993); Spect. 24.2 (Turcan 1986). Cf. Verheijen 1957; Michaélidès 1970, 43-45, 166-167, 178-179, 260-262. Queste parole si trovano anche nelle opere di Cipriano, ma si usano spesso in relazione al segno di croce postbattesimale: Ad Demetr. 22.10 (Gallicert - Veronese 2018); Ep. 73.9.2 (Diercks 1996); Laps. 2 (Bévenot 1972). Cf. Reijner 1965, 162-167; Gallicert - Veronese 2018, 277 (commento a 22.10).

58 Ambr. Symb. 1 (Botte 1994): Nunc tempus est et dies ut symbolum tradamus, quod symbolum est spiritale signaculum, quod symbolum cordis est nostri meditatio et quasi semper praesens custodia, certe thesaurus pectoris nostri (trad. Compagnoni 2013, 37).

59 Rufin. Apol. adv. Hier. 1.4 (Simonetti 1961): Ego, sicut et ipse et omnes norunt, ante annos fere triginta in monasterio iam positus, per gratiam baptismi regeneratus, signaculum fidei consecutus sum per sanctos viros Chromatium Iovinum et Eusebium (trad. Simonetti 1999, 85).

60 Rufin. Apol. adv. Hier. 1.4 (Simonetti 1961): pater mihi et doctor symboli ac fidei fuit.

61 Kinzig 2017, 1.6.
} 


\subsection{Confronto con il Simbolo degli Apostoli}

Com'è il Simbolo priscillianista? Il Simbolo di Priscilliano è più simile al Simbolo degli Apostoli che al Simbolo niceno, essendo il primo il simbolo battesimale conosciuto bene in Occidente in quel tempo. È opinione diffusa che il testo attuale del Simbolo sia comparso alla fine del VII secolo, ma, nonostante ciò, esistevano il Simbolo Romano, alla base di questo Simbolo, e le sue varianti nel quarto secolo e 1'archetipo greco di questi Simboli. ${ }^{62}$

Seguendo L. Westra, chiamo Simbolo degli Apostoli non solo il simbolo che ha il testo attuale ma anche i simboli che sono varianti dell'originale e di cui un rappresentante è il Simbolo Romano. Infatti, prima della diffusione del testo attuale, il Simbolo degli Apostoli, che gli autori ecclesiastici hanno usato, non era unico ma era diffuso in numerose varianti in quanto i testi hanno raramente coinciso, differenziandosi non solo per le espressioni e la struttura ma anche per il contenuto. ${ }^{63}$ Eppure ci sono delle differenze non trascurabili nel confronto con il Simbolo di Priscilliano. Qui mostro il testo del Simbolo Romano come punto di riferimento e lo confronto con il simbolo di Priscilliano. ${ }^{64}$

\section{Roma}

1 Credo in deum patrem omnipotentem

2 et in Christum Iesum, filium eius unicum dominum nostrum

3 qui natus est de spiritu sancto et Maria virgine

4 qui sub Pontio Pilato crucifixus est

5 et sepultus tertia die resurrexit a mortuis

6 ascendit in caelos sedet ad dexteram patris

7 Unde venturus est iudicare vivos et mortuos

8 et in spiritum sanctum, sanctam ecclesiam

9 remissionem peccatorum

10 carnis resurrectionem

\section{Priscilliano}

Credentes unum deum patrem omnipotentem

et unum dominum Iesum Christum

natum ex Maria virgine ex spiritu sancto

Passum sub Pontio Pilato, crucifixum

sepultum, tertia die resurrexisse

ascendisse in caelos, sedere ad dexteram dei patris omnipotentis

inde venturum et iudicaturum de vivis et mortuis credentes in sanctam eclesiam, sanctum spiritum, baptismum salutare

credentes remissionem peccatorum

credentes in resurrectionem carnis

Le caratteristiche peculiari del simbolo di Priscilliano sono:

(a) l'aggiunta di unus nell'art. 1 .

(b) l'aggiunta di unus e l'omissione di filius eius unicus nell'art. 2.

(c) l'inversione nell'ordine fra Spirito Santo e Vergine Maria nell'art. 3.

62 Kelly 1972, 102-130; Westra 2002, 21-72; Kinzig 2017, 1.12-13.

63 Westra 2002, 76-78. La differenza dell'espressione e della struttura è, per esempio, la divergenza tra ad caelum e in caelos nell'art. 6, e la diversità tra la proposizione con qui e il complemento oggetto negli art. 3 e 4 . La differenza del contenuto è, ad esempio, la presenza di creatorem caeli et terrae nell'art. 1 (non c'è nel Simbolo Romano, ma nel testo attuale) e della frase descendit ad inferna dopo la sepoltura nell'art. 5 (non c'è nel Simbolo Romano, ma nel testo attuale). Cf. Rufin. Symb. 16 (Simonetti 1961) (Simbolo di Aquileia).

${ }_{64}$ Westra $(2002,99-276)$ ha analizzato tutti i tipi del Simbolo degli Apostoli e li ha confrontati. Questo testo riproduce quello di Westra 2002, 27, ma l'articolazione si fa corrispondere a Priscilliano per convenienza di confronto. Riguardo alla articolazione generale del simbolo: Westra 2002, 11-12. 
(d) l'inversione nell'ordine tra Spirito Santo e Santa Chiesa nell'art. 8.

(e) l'aggiunta di baptismum salutare nell'art. 8.

Nonostante ciò, la maggior parte di queste peculiarità si può spiegare come riflessione della situazione della Chiesa in Hispania o come influsso di qualche esempio.

Riguardo alle differenze di (a) e (b), Toom ha proposto un esame dettagliato. ${ }^{65}$ In primo luogo, è notevole che siano riprese le citazioni da I Cor. 8.6 nell'art. 1 e 2 . Considerata la presenza delle citazioni bibliche, si potrebbe concludere che queste varianti seguano la Bibbia. In secondo luogo, l'aggiunta di unus a Padre e a Figlio è usuale in Oriente. Un buon esempio è il Simbolo niceno in cui ci sono "un solo Dio

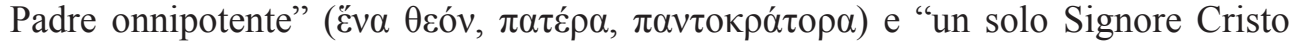

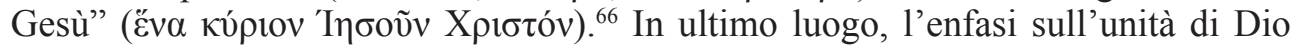
non è estranea in Spagna durante la seconda metà del quarto secolo. Si diceva che Gregorio di Elvira fosse il capo dei luciferiani dopo la morte di Lucifero di Cagliari, e che fosse accusato di Monarchianismo, ${ }^{67}$ in seguito negli anni 383/384, due sacerdoti luciferiani fecero una petizione all'imperatore Teodosio contro l'accusa di Monarchianismo e testimoniarono che i loro compagni avevano patito un attacco in Hispania. ${ }^{6}$ Insomma, oltre ai priscillianisti c'erano in Spagna molti difensori dell'unità divina che avrebbero potuto essere accusati di Monarchianismo.

Anche l'aggiunta del battesimo in (e) si potrebbe comprendere in relazione alla situazione in quel tempo. Dopo la morte di Damaso, il suo successore Siricio per prima cosa contestò il secondo battesimo per gli ex-ariani nella risposta a Imerio, vescovo di Tarragona che aveva consultato la sede apostolica sui problemi nelle chiese spagnole. ${ }^{69} \mathrm{Si}$ potrebbe ipotizzare che in Spagna sia stata viva la controversia sull'efficacia del battesimo dall'eresia, e che l'aggiunta della menzione del battesimo nel Simbolo rifletta questa disputa.

Al contrario, non si possono spiegare bene le caratteristiche (c) e (d). In entrambi lo Spirito Santo è posto dopo, pertanto si potrebbe concludere che Priscilliano ha sottovaluto questa persona. Ma in realtà sia nel Liber apologeticus sia nel Liber ad Damasum Priscilliano insiste su tre persone, quando afferma "l'indissolubile fede che promana da unica fonte tripartita"70 e "battezzando, com'è scritto, nel nome del Padre, del Figlio e dello Spirito Santo". ${ }^{71}$ E anche per (d) si potrebbe dire che Priscilliano ha sottolineato l'ecclesia sancta perché ha pensato che la chiesa si deve costruire con le genti pure. ${ }^{72}$ Tutto sommato, le caratteristiche del Simbolo di Priscilliano si possono collocare in qualche misura nella situazione teologica coeva. Tuttavia Priscilliano non ha fondamentalmente considerato queste caratteristiche come differenze rispetto al testo diffuso. Brevemente provo ora a illustrare alcuni esempi di scrittori coevi che presentano varianti nel testo del Simbolo secondo la spiegazione di Westra. ${ }^{73}$

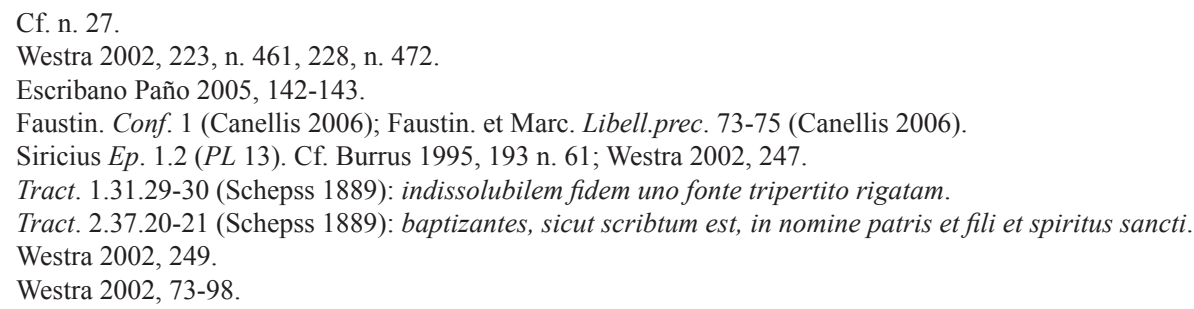


Su questa questione lo studioso ha presentato le testimonianze di Rufino e di Agostino come buon esempio che dimostra come gli ecclesiastici comprendono le differenze tra i testi del Simbolo degli Apostoli. Per primo Rufino nella Expositio symboli parla di tre simboli battesimali (Aquileisese, Romano, Orientale) e fa riferimento alle differenze tra loro. Benché apprezzi il Simbolo Romano comel'origine dei simboli, l'Aquileiese conferisce autorità anche agli altri simboli, considerando che le differenze siano state introdotte per due ragioni: o per controbattere l'eresia ${ }^{74}$ oppure perché fondate sulla Bibbia. ${ }^{75}$ Pertanto si può dire che Rufino ha accettato tutti e tre i Simboli malgrado non presentino lo stesso testo.

Si legge una possibilità più audace dalle testimonianze di Agostino, il quale cita il Simbolo in diverse opere, ma sembra che usi due simboli differenti nel contenuto. Generalmente si ritiene che l'uno sia il Simbolo di Milano che Agostino stesso ha imparato quando ha ricevuto il battesimo e che l'altro sia il Simbolo tradizionale della chiesa di Ippona.

\begin{aligned} & \multicolumn{1}{c}{ Milano $^{76}$} \\ 1 & Credo in deum patrem omnipotentem \\ 2 & et in Christum Iesum, filium eius unicum dominum \\ & nostrum \\ 3 & qui natus est de spiritu sancto et virgine Maria \\ 4 & sub Pontio Pilato crucifixus \\ 5 & et sepultus, tertia die resurrexit a mortuis \\ 6 & ascendit in caelum sedet ad dexteram patris \\ 7 & inde venturus iudicaturus vivos et mortuos \\ 8 & et in spiritum sanctum, sanctam ecclesiam \\ 9 & remissionem peccatorum \\ 10 & carnis resurrectionem \\ 11 & \end{aligned}

\author{
Ippona $^{77}$ \\ Credo in deum patrem omnipotentem, universorum \\ creatorem, regem saeculorum, immortalem et invisi- \\ bilem \\ (credo?) et in filium eius Iesum Christum, dominum \\ nostrum \\ natum de spiritu sancto et virgine Maria \\ crucifixum sub Pontio Pilato \\ et sepultum tertia die a mortuis resurrexit \\ ascendit in caelum sedet ad dexteram patris \\ inde venturus est iudicare vivos et mortuos \\ (credo?) (et?) in spiritum sanctum \\ [remissionem peccatorum] \\ [resurrectionem carnis] \\ in vitam aeternam per sanctam ecclesiam
}

C'è una grande differenza tra i due Simboli dopo l'art. 8. Nel Simbolo di Milano si dice: "credo nello Spirito Santo, la santa Chiesa", mentre "la santa Chiesa" nel Simbolo di Ippona non è oggetto di credo nell'art. 8, ma si trova a-lla fine come "per la santa Chiesa". Agostino non ha detto niente su questa differenza, ma gli studiosi ipotizzano che abbia usato il secondo Simbolo in forma privata e il primo nel servizio pastorale a Ippona rispettando la tradizione locale. ${ }^{78}$ Ma pure apre ad un'altra possibilità il Sermo 214 che fu pronunciato davanti alla comunità di Ippona

\footnotetext{
74 Rufin. Symb. 5 (Simonetti 1961).

75 Rufin. Symb. 16 (Simonetti 1961).

76 Il testo del Simbolo in Agostino e negli altri scrittori antichi, generalmente non è riportato integralmente, ma si dispiega passo dopo passo integrato da spiegazioni e commenti. Il testo che riporto qui è quello citato da Westra 2002, 189-196. L'articolazione si fa corrispondere a Priscilliano per convenienza di confronto. Cito qui anche i testi originali del Serm. 213 (Morin 1930) e del Symb. (Vander Plaetse 1969). Il numero in parentesi quadre indica il capitolo in cui si trova la frase.

77 Serm. 215 (Verbraken 1958). Westra 2002, 163-168.

78 Kinzig 2017, 2.303.
} 
per la preparazione al battesimo, e in cui Agostino spiega genericamente il Simbolo, dichiara di procedere alla lettura dello stesso, che tuttavia è omesso nel testo dell'omelia, esattamente come accade anche negli altri sermoni, e infine dispiega l'interpretazione di ciascuna frase. È importante il fatto che il testo base è quello del Simbolo di Milano, nonostante il sermone sia stato tenuto a Ippona. Da questo Westra ha concluso che Agostino considera entrambi come il Simbolo degli Apostoli, malgrado le differenze di testo. ${ }^{79}$ Tutto sommato, non è stato strano identificare i due testi differenti pensando che entrambi dimostrino la dottrina ortodossa, nonostante sia un altro problema come si comprende questo comportamento.

Si dice pertanto che il simbolo di Priscilliano è uno di tipi del Simbolo degli Apostoli nel quarto secolo, pur essendo differente dal testo attuale, ma riflettendo la situazione teologica contemporanea nel contenuto. Non solo Priscilliano ma anche altri autori tra la fine del IV e l'inizio del V secolo, come Westra ha dimostrato, sosteneva che il suo Simbolo fosse il simbolo ortodosso trasmesso per tradizione da Cristo e dagli Apostoli tollerando varianti.

\subsection{Simbolo degli Apostoli e Simbolo niceno nella Chiesa occidentale durante la seconda metà del quarto secolo}

Priscilliano si è difeso dalle accuse con il Simbolo degli Apostoli, ma perché ha usato questo simbolo benché fosse passato più di mezzo secolo dal Concilio niceno e la fede del Simbolo niceno stesse per stabilirsi come la dottrina ortodossa? In Oriente, dopo il Concilio niceno nel 325, si provò a correggere o a completare il Simbolo niceno oppure a riconfermarlo, e le dichiarazioni contenenti i simboli si confrontarono in numerosi concili sulle varie posizioni teologiche, riguardo al termine "omousia". D'altra parte, in Occidente si aprirono dopo la metà del IV secolo i concili sul problema trinitario, su cui influì Costanzo II che fu un grande sostenitore degli antiniceni. La chiesa occidentale rimase fedele alla sua conoscenza di Dio e alla dottrina del Simbolo niceno e non si impegnò nella disputa sul Simbolo.

Tuttavia si potrebbe dire che gli occidentali condividano il contenuto teologico del Simbolo niceno, anche se continuano a utilizzare il Simbolo degli Apostoli e

79 Westra (2002, 85, n. 43) indica anche sei esempi che prima presentano un testo o un nome di simbolo e dopo espongono un altro testo oppure identificano un Simbolo con l'altro: Caes.Arel. Serm. 9 (Morin 1953); Miss.Bob. 183-185 (Lowe 1920); Ps. Facund. Fid. 14; 20 (Clément - Vander Plaetse 1974), su cui cf. Fraïsse - Bétoulières 2006, 277-282, la quale dimostra che questa opera è pseudepigrafa; Faust. Rei. Spir. 1.1 (Engelbrecht 1891); Vigil.Thapens. C.Eutych. 4.1 (Petri 2003); Cassian. C.Nest. 4.1 (Petschenig - Kreuz 2004). Ma per quasi tutti gli esempi manca la sicurezza del testo o è presente solo qualche sfumatura. Per primo, Serm. 9 di Cesario e Miss.Bob. testimoniano la presenza di due versioni diverse nello stesso testo; ma ciò dipende dall'intervento di un redattore successivo che ha aggiunto all'inizio dei due scritti il testo del Simbolo diffuso ai suoi tempi, che si differenzia rispetto al testo commentato dall'autore antico. Cf. Westra 2002, 121-127, 350. Per secondo, Westra sostiene che Facondo, Fausto e Vigilio hanno considerato il Simbolo niceno-costantinopolitano e il Simbolo degli Apostoli come lo stesso "Simbolo". Ma in Facondo (Fid. 14) e Vigilio (C.Eutych. 4.1) si indica che il primo è la forma ampliata del secondo che costituisce l'originale. Una testimonianza che mi bisogna è che l'autore considera i due testi coevi come lo stesso del Simbolo. A questa condizione mi è utile solo Cassian. C.Nest. In questa opera Cassiano mostra "il testo del Simbolo antiocheno" (textus... Antiocheni symboli) che è più simile al Simbolo niceno-costantinopolitano che al Simbolo degli Apostoli (4.3), e considera il Simbolo antiocheno come il Simbolo tradotto dagli Apostoli (4.4) e il Simbolo battesimale (4.5). E chiama questo Simbolo esattamente "il Simbolo antiocheno", non "il Simbolo", non "il Simbolo niceno". Da questo mi sembra di poter concludere che Cassiano abbia intenzionalmente ignorato le differenze fra il testo del Simbolo antiocheno e quello diffuso in Gallia. 
le sue varianti, come dimostra il fatto che il Simbolo niceno faccia la sua prima comparsa in Occidente solo dopo la metà del IV.$^{80}$ Benché la mancanza di fonti non significhi che il Simbolo niceno fosse sconosciuto fino a quel tempo, tuttavia è molto interessante la testimonianza di Ilario di Poitiers, per il quale la fedeltà alla fede nicena non necessariamente significa l'uso del Simbolo niceno:

Tuttavia in queste cose, tanto beati nel Signore e gloriosi voi che, ritenendo la fede perfetta e apostolica con la professione coscienziosa, fino ad ora ignoravate delle fedi scritte! Infatti non occorreva la lettera a voi che abbondavate dello spirito. Neanche desideravate un lavoro manuale per scrivere, voi che ciò che credevate di cuore confessavate con la bocca per la salvezza. Neppure voi vescovi avevate bisogno di leggere quello che tenevate rigenerati come neofiti. Tuttavia si impone la necessità di produrre la consuetudine di esporre le fedi e di sottoscrivere quanto esposto. Dove la comprensione di fede è in pericolo, allora si richiede la lettera. Non si impedisce di scrivere ciò che è salutare confessare. ${ }^{81}$

Essendo stato battezzato da tempo ed essendo vescovo da un po'di tempo, non avevo mai sentito della fede nicena prima dell'esilio. Ma il Vangelo e gli Apostoli mi avevano insegnato il significato di homousios e di homoeusios. ${ }^{82}$

Da queste notizie deduciamo che fosse estranea all'Occidente alla metà del quarto secolo la tradizione di definire il Simbolo in un concilio, come invece accaduto in Oriente al Concilio di Nicea. Questa ipotesi è sostenuta dalla testimonianza di Marcello di Ancira, un rigorista antiariano nel Concilio niceno, deposto dall'episcopato nella controversia e condannato all'esilio due volte. Partendo per Roma nel 338/9 durante il suo secondo esilio, dovette esporre la sua fede a Papa Liberio con una lettera per farsi accettare. In questa lettera, pervenutaci attraverso il Panarion di Epifanio, la professione di Marcello è consistente non nel Simbolo niceno ma nel Simbolo degli Apostoli. ${ }^{83}$ Analogamente in Occidente alla metà del quarto secolo è più comune il Simbolo degli Apostoli che il Simbolo niceno. McLynn sostiene che Priscilliano aveva mostrato il carattere locale della chiesa spagnola utilizzando un simbolo locale piuttosto che il Simbolo niceno più generale. ${ }^{84}$ Tuttavia penso che il simbolo di Priscilliano sia il Simbolo degli Apostoli e che l'uso di questo si debba comprendere come un esempio che mostra la continuità della situazione della metà del quarto secolo.

80 Liber. Ep. ad Const. 132-160 (Diercks 1978) è antichissimo, ma si dubita dell'autenticità. È più sicuro Hil. Ad Const. 1.8.2-3 (=coll. antiar. app. 2.3.2-3) (Feder 1916). Cf. Kinzig 2002, 1.13, n. 60.

81 Syn. 63 (PL 10): Sed inter haec, o beatos vos in Domino et gloriosos, qui perfectam atque apostolicam fidem conscientiae professione retinentes, conscriptas fides huc usque nescitis! Non enim eguistis littera, qui spiritu abundabatis. Neque officium manus ad scribendum desiderastis, qui quod corde a vobis credebatur, ore ad salutem profitebamini. Nec necessarium habuistis episcopi legere, quod regenerati neophyti tenebatis. Sed necessitas consuetudinem intulit, exponi fides, et expositis subscribi. Ubi enim sensus conscientiae periclitatur, illic littera postulatur. Nec sane scribi impedit, quod salutare est confiteri.

82 Syn. 91 (PL 10): Regeneratus pridem, et in episcopatu aliquantisper manens, fidem Nicaenam numquam nisi exsulaturus audiui: sed mihi homousii et homoeusii intelligentiam Euangelia et Apostoli intimaverunt.

83 Epiph.Const. Haer. 72.2.6-3.4 (Holl - Dummer 1985).

84 McLynn 1997, 175. 


\section{Conclusione}

Priscilliano fonda la sua ortodossia sulla base di un Simbolo che è sicuramente differente dal Simbolo niceno. Considerato che Priscilliano fu condannato per eresia, il suo Simbolo si caratterizzava come una imitazione e non era riflesso a fondo il suo uso di quello. In questi decenni, tuttavia, i ricercatori cercano di disporre Priscilliano nel contesto coevo distanziando la dicotomia ortodossaeresia, e anche questo articolo prova a rivalutarlo dal punto di vista del Simbolo. In primo luogo, rifletto su quello che Priscilliano ci pensava e dimostro che non fu strana la sua insistenza sul Simbolo mettendola a confronto degli esempi coevi e della tradizione nella Chiesa occidentale. In secondo luogo, dipendente dai precedenti studi analizzando il testo del Simbolo di Priscilliano e dando gli esempi che i Simboli erano considerati come lo stesso nonostante le differenze di testo, confermo che in quel tempo il Simbolo di Priscilliano si può comprendere come il Simbolo degli Apostoli. Alla fine, proposto il significato dell'uso del Simbolo da Priscilliano tendendo in considerazione il riconoscimento del Simbolo niceno in occidentale a quel tempo. In somma, il Simbolo di Priscilliano è un'importante testimonianza per capire la realtà del cristianesimo nella chiesa occidentale durante la seconda metà del quarto secolo, perché il suo Simbolo è una chiave per interpretare le caratteristiche del suo testo e la preferenza per il Simbolo degli Apostoli come elementi locali in Hispania.

\section{Bibliografia}

Babut, E.-Ch. (1909): Priscillien et le priscillianisme (=Bibliothèque de l'École des Hautes Études 169), Paris.

Barbero de Aguilera, A. (1963): “El Priscilianismo: ¿herejía o movimiento social?”, Cuadernos de Historia de España 37-38, 5-41.

Blaise, A. (1967): Dictionnaire latin-français des auteurs chrétiens, Turnhout.

Borleffs, J. G. Ph. (ed.), (1954): De baptismo, in Quinti Septimi Florentis Tertulliani opera, pars I (=CCSL 1), Turnhout.

Botte, B. (ed. \& tr.), (1994): Ambroise de Milan. Des sacrements. Des mystères. Explication du symbole (=SC 25bis), Paris.

Braun, R. (ed. \& tr.), (1990): Tertullien. Contre Marcion, livre I (=SC 365), Paris.

Burrus, V. (1995): The Making of a Heretic: Gender, Authority, and the Priscillianist Controversy (=The Transformation of the Classical Heritage 24), Berkeley-Los Angeles-London.

Canellis, A. (ed. \& tr.), (2006): Faustin et Marcellin. Supplique aux empereurs (=SC 504), Paris. Carnandet, J. (ed.), (1867): Acta Sanctorum: Junii tomus quartus, Paris.

Clément, I. M. - Vander Plaetse, R. (eds.), (1974): Facundi episcopi ecclesiae Hermianensis opera omnia $(=$ CCSL 90A), Turnhout.

Compagnoni, G. M. (tr.), (2013): Sant'Ambrogio. La luce nel cuore: catechesi sulla fede, Milano. Conti, M. (2010): Priscillian of Avila: The Complete Works, Oxford.

Chadwick, H. (1976): Priscillian of Avila: The Occult and the Charismatic in the Early Church, Oxford.

Daur, K-D. (ed.), (1985): Aurelii Augustini opera, pars XV, 3 (=CCSL 49), Turnhout.

Diercks, G. F. (ed.)

(1978): Luciferi Calaritani opera quae supersunt (=CCSL 8), Turnhout. 
(1996): Sancti Cypriani episcopi epistularium: Cyprianus epistulae 58-81 (=CCSL 3 C), Turnhout.

De Senneville-Grave, G. (ed. \& tr.), (1999): Sulpice Sévère. Chroniques (=SC 441), Paris.

Engelbrecht, A. (ed.), (1891): Fausti Reiensis praeter sermones pseudo-eusebianos opera $(=$ CSEL 21), Wien.

Escribano Paño, M $M^{\mathrm{a}}$ V. (2005): "Heresy and Orthodoxy in Fourth Century Hispania: Arianism and Priscillianism", [en] K. Bowes - M. Kulikowski (eds. e trs.), Hispania in Late Antiquity: Current Perspectives (=The Medieval and Early Modern Iberian World 24), Leiden-Boston, 121-149.

Feder, A. (ed.), (1916): S. Hilarii episcopi Pictaviensis opera, pars quarta: Tractatus mysteriorum, Collectanea antiariana parisina (Fragmenta historica) cum appendice (Liber I ad Constantium), Liber ad Constantium imperatorem (Liber II ad Constantium), Hymni, Fragmenta minora, Spuria (=CSEL 65), Wien.

Fraïsse-Bétoulières, A. (tr.), (2006): Facundus d'Hermiane. Défense des trois chapitres (à Justinien), tome IV (Livres XI-XII), (=SC 499), Paris.

Gaffiot, F. (ed.) - Flobert, P. (rev.), (2000): Le Grand Gaffiot Dictionnaire Latin-Français, Paris.

Gallicert, E. - Veronese, M. (ed. \& tr.), (2018): Cipriano. A Demetriano, Torino.

Gams, P. (1864): Die Kirchengeschichte von Spanien, Bd. 2.1, Regensburg.

Hayashi, K. (2018): "Priscillian of Avila and his Creed", Patristica: Proceedings of the Colloquia of the Japanese Society for Patristic Studies 22, 19-40 [in giapponese].

Hilberg, I. - Kamptner, M. (eds.), (1996): Sancti Eusebii Hieronymi Epistulae pars III: Epistulae CXXI-XLIV (=CSEL 56.1.3), Wien.

Hodgers, R. H. (ed.), (1975): Palladii Rutilii Tauri Aemiliani viri inlustris Opus agriculturae. De veterinaria medicina. De insitione, Leipzig (https://doi.org/10.1515/9783110966206).

Holl, K. - Dummer, J. (eds.), (1985): Epiphanius III: Panarion haer. 65-80, De fide (=GCS 37), Berlin.

Kelly, J. N. D. (1972): Early Christian Creeds, New York.

Kinzig, W.

(2011): "The Creed and the Development of the Liturgical Year in the Early Church", [en] J. Dochhorn (ed.), "For It Is Written": Essays on the Function of Scripture in Early Judaism and Christianity, Frankfurt a.M., 63-99 (https://doi.org/10.3726/978-3-653-01624-6).

(2017): Faith in Formulae. A Collection of Early Christian Creeds and Creed-related Texts, vol. 1-4, Oxford.

Kübler, B. - Seckel, E. (eds.), (1908): Iurisprudentiae anteiustinianae reliquias, Leipzig.

Künstle, K. (1905): Antipriscilliana. Dogmengeschichtliche Untersuchungen und Texte aus dem Streite gegen Priscillians Irrlehre, Freiburg-im-Breisgau.

Lowe, E. A. (ed.), (1920): The Bobbio Missal: A Gallican Mass-Book (MS. PARIS. LAT. 13246), London.

Matthews, J. (1975): Western Aristocracies and Imperial Court: AD 364-425, Oxford.

McLynn, N. (1997): “Theodosius, Spain and the Nicene Faith”, [en] R. Teja - C. Pérez (eds.), Congreso Internacional: La Hispania de Teodosio (Segovia-Coca. Octubre, 1995), Salamanca, 171-178.

Mommsen, T. (ed.) - Rougé, J. (tr.), (2005): Les lois religieuses des empereurs romains de Constantin à Théodose II (312-438), vol. 1 (=SC 497), Paris.

Michaélidès, D. (1970): "Sacramentum” chez Tertullien, Paris.

Moreschini, C. (ed.) - Braun, R (tr.), (2004): Tertullien. Contre Marcion, libre V (=SC 483), Paris.

Morin, G. (ed.) 
(1930): Sancti Augustini Sermones post Maurinos reperti, Roma.

(1953): Sancti Caesarii Arelatensis Sermones, I (=CCSL 103), Turnhout.

Munier, C. (ed. \& tr.)

(1984): Tertullien. La pénitence (=SC 316), Paris.

(1993): Tertullien. La pudicité, 1 (=SC 394), Paris.

Niermeyer, J. F. - Van de Kieft, C. (eds.), (2002): Mediae Latinitatis Lexicon Minus, Leiden (rev. e tr. J. W. J. Burgers).

Orbe, A. (1981): "Heterodoxia del [Priscilliani] Tractatus Genesis", Hispania Sacra 33, $285-$ 311.

Petri, S. (ed. \& tr.), (2003): Vigilio di Tapso. Contro Eutiche, Brescia.

Pietri, C. - Pietri, L. (eds.), (2000): Prosopographie de l'Italie chrétienne (313-604), vol. 2, Roma.

Refoulé, R. F. (ed.) - De Labriolle, P. (tr.), (1957): Tertullien. Traité de la prescription contre les hérétiques (=SC 46), Paris.

Reijner, C. Q. (1965): The Terminology of the Holy Cross in Early Christian Literature as Based upon Old Testament Typology (=GCP 2), Nijmegen.

Richardson, E. C. (ed.), (1896): Hieronymus liber de viris inlustribus. Gennadius liber de viris inlustribus (=TU 14.1), Leipzig.

Sanchez, S. J. G. (2009): Priscillien, un Chrétien non conformiste. Doctrine et pratique du priscillianisme du $\mathrm{IV}^{e}$ au $V I^{e}$ siècle (=Théologie historique 120), Paris.

Schepss, G. (1889): Priscilliani quae supersunt (=CSEL 18), Wien.

Schipper, H. G. - Van Oort, J. (eds. \& trs.), (2000): St. Leo the Great. Sermons and Letters against the Manichaeans. Selected Fragments (=Corpus Fontium Manichaeorum Series Latina 1), Turnhout.

Simonetti, M. (ed.)

(1961): Tyrannius Rufinus. Opera (=CCSL 20), Turnhout.

(1999): Rufino di Concordia. Scritti apologetici, Roma.

Stroheker, K. F. (1963): "Spanische Senatoren der spätrömischen und westgothischen Zeit", Madrider Mitteilungen 4, 107-132.

Toom, T.

(2014a): "Marcellus of Ancyra and Priscillian of Avila: Their Theologies and Creeds", Vigiliae Christianae 68, 60-81 (https://doi.org/10.1163/15700720-12341159).

(2014b): “Was Priscillian a Modalist Monarchian?", Harvard Theological Review 107, 470484 (https://doi.org/10.1017/S0017816014000376).

Turcan, M. (ed. \& tr.), (1986): Tertullien. Les spectacles (=SC 332), Paris.

Vander Plaetse, R. (1969): “Sermo de símbolo ad catechumenos", [en] Sancti Aurelii Augustini De fide rerum invisibilium. Enchiridion ad laurentium de fide et spe et caritate. De catechizandis rudibus. Sermo ad catechumnos de símbolo. Sermo de disciplina christiana. Sermo de utilitate ieiunii. Sermo de excidio urbis Romae. De haeresibus (=CCSL 46), Turnhout, 185-199.

Van Waarden, J. (2014): "Priscillian of Avila's Liber ad Damasum and the Inability to Handle a Conflict", [en] A. C. Geljon - R. Roukema (eds.), Violence in Ancient Christianity: Victims and Perpetrators (=Vigiliae Christianae, Suppl. 125), Leiden, 132-150 (https://doi.org/10. 1163/9789004274907_009).

Verbraken, P. (1958): "Les sermons CCXV et LVI de Saint Augustin, De symbolo et De oratione dominica", Revue Bénédictine 68, 5-40 (https://doi.org/10.1484/J.RB.4.01462).

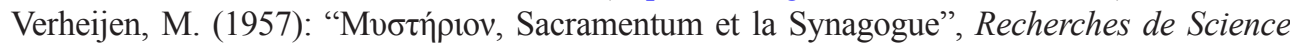
Religieuse 45, 321-337.

Vollmann, B. K. (1974): "Priscillianus", [en] Paulys Realencyclopädie der classischen Altertumswissenschaft, Supplementband XIV, München, 485-559. 
Waszink, J. H. - Van Winden, J. C. M. (eds. \& trs.), (1987): Tertullianus. De idolatria (=Vigiliae Christianae, Suppl. 1), Leiden-New York (https://doi.org/10.1163/9789004312715).

Westra, L. H.

(2002): The Apostle' Creed: Origin, History, and Some Early Commentaries, Turnhout. (2010): “How Did Symbolum Come to Mean 'Creed'?", Studia Patristica 45, 85-91.

Zycha, I. (ed.), (1900): Sancti Aureli Augustini De fide et symbol. De fide et operibus. De agone christiano. De continentia. De bono coniugali. De sancta virginitate. De bono viduitatis. De adulterinis coniugiis. lib. II De mendacio. Contra mendacium. De opere monachorum. De divinatione daemonum. De cura pro mortuis gerenda. De patientia (=CSEL 41), Wien. 